\title{
ON IMPLEMENTATION AND DESIGN OF FILTER BANKS FOR SUBBAND ADAPTIVE SYSTEMS
}

\author{
S. Weiß, M. Harteneck, and R.W. Stewart \\ Signal Processing Division, Dept. of Electronic and Electrical Eng. \\ University of Strathclyde, Glasgow, Scotland \\ \{weiss, moritz, bob\}@spd.eee.strath.ac.uk
}

\begin{abstract}
In this paper, we introduce a polyphase implementation and design of an oversampled $K$-channel generalized DFT (GDFT) filter bank, which can be employed for subband adaptive filtering, and therefore is required to have a low aliasing level in the subband signals. A polyphase structure is derived which can be factorized into a real valued polyphase network and a GDFT modulation. For the latter, an FFT realization may be used, yielding a very inexpensive polyphase implementation for arbitrary integer decimation ratios $N \leq K$. We also present an analysis underlining the efficiency of complex valued subband processing. The design of the filter bank is completely based on the prototype filter and solved using a fast converging iterative least squares method, for which we give examples. The design specifications closely correspond with performance limits of subband adaptive filtering, which are under-pinned by simulation results.
\end{abstract}

\section{INTRODUCTION}

Subband adaptive filter (SAF) systems, as shown in Fig. 1 for a system identification setup, are widely used for problems like acoustic echo cancellation (AEC), where an adaptive system is required to identify very long impulse responses, which becomes viable due to decreased complexity by processing in decimated subbands. However, the case of critical decimation, where the decimation ratio $N$ equals the number of uniform subbands $K$, requires either cross-terms at least between adjacent frequency bands [3], which compensates for the information loss in the region of spectral overlap, or gap filter banks $[17,12]$, which introduces spectral loss that may not be acceptable.

Oversampled SAF systems can be either real or complex valued. Real valued bandpass signals have to be modulated into the baseband prior to decimation by, for example, single sideband modulation (SSB, $[1,14]$ ), or their bandwidth and decimation ratio has to be chosen in accordance with the sampling theorem, leading to non-uniform filter banks $[11,5]$. In contrast, the decimation of complex valued bandpass signals with any integer factor $N<K$ is straightforward. 


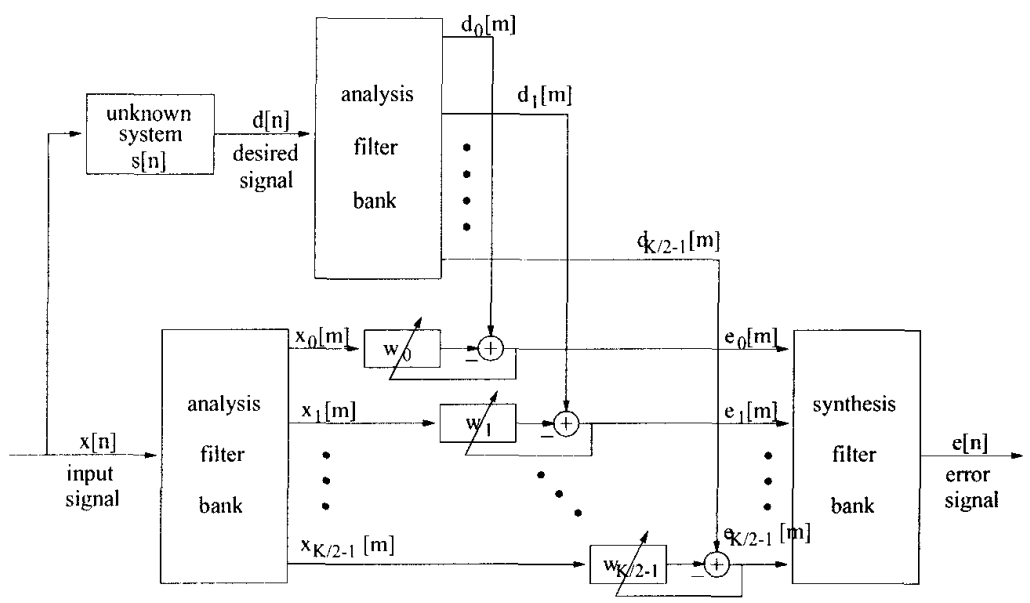

Fig. 1: Subband adaptive filter structure in a system identification setup.

In this paper, we will focus on generalized DFT filter banks [1], which perform a particular type of complex valued subband decomposition. GDFT filter banks arise from complex modulation of a prototype filter, and, as will be derived, can be very efficiently realized using for generally non-integer oversampling ratios $K / N$. This will prove an often stated misconception wrong that polyphase implementations are only viable for integer oversampling ratios (OSR) $\frac{N}{K} \in \mathbb{Z}[1,8]$, while otherwise frequency domain realizations of the filter banks are preferred [10]. This is particularly important, since subband processing shows its highest reduction in computational complexity for OSRs close to 1 .

Further, we will adopt an iterative least squares method method $[9,6]$ to design prototype lowpass filters for GDFT modulated oversampled filter banks appropriate for SAF systems. The error performance of the produced SAFs can be linked to the design criteria of this prototype filter [15]. Finally, we will present some prototype designs, which will be used in adaptive system identification examples performed in subbands.

\section{COMPLEX SUBBAND DECOMPOSITION BY GDFT FILTER BANKS}

GDFT Modulation. A general structure of a $K$ channel filter bank with decimation by a factor $N \leq K$ is shown in Fig. 2. The analysis filters $h_{k}[n]$ are derived from a real valued lowpass prototype FIR filter $p[n]$ of even length $L_{p}$ by a generalized discrete Fourier transform (GDFT),

$$
h_{k}[n]=e^{j \frac{2 \pi}{K}\left(k+k_{0}\right)\left(n+n_{0}\right)} \cdot p[n], \quad k, n \in \mathbb{N},
$$

which can be closely linked to a DCT-IV modulation used for cosine modulated pseudo-QMF $K / 2$ channel filter banks [13] by a real operation performed 


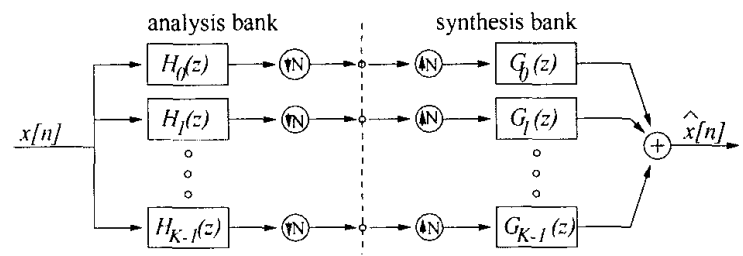

Fig. 2: Analysis and synthesis branch of a $K$-channel filter bank with subbands decimated by $N$.

on the complex bandpass filters $h_{k}[n]$.

The term generalized DFT [1] stems from offsets $k_{0}$ and $n_{0}$ introduced into the frequency and time indices in the modulation term of (1). Linear phase property of the modulated filters can be ensured by choosing a linear phase prototype filter $p[n]$ and a transform symmetric to $\left(L_{p}-1\right) / 2$ by appropriately setting $n_{0}$. A frequency offset $k_{0}=\frac{1}{2}$ shifts the bandpass characteristics of the filters $h_{k}[n]$ and yields the frequency range $[0 ; \pi]$ to be covered by exactly $K / 2$ subbands for an even $K$, while the remaining subbands are complex conjugate versions and therefore redundant to process, if the input signal to the filter bank is real.

The synthesis filters $g_{k}[n]$ can be obtained by time reversion of the analysis filter, i.e. $g_{k}[n]=\tilde{h}_{k}[n]=h_{k}^{*}\left[L_{p}-n+1\right]$. Thus, all filters can be derived from one single prototype $p[n]$, which has to be designed appropriately.

\section{Efficient Filter Bank Implementation}

For efficient implementation of the oversampled GDFT filter bank, we employ polyphase representation of the analysis and synthesis filters. Generally, savings due to a polyphase implementation are gained in two steps: firstly, the calculation of decimated samples will be suppressed; secondly, computations common to different branches of the analysis or synthesis bank are combined.

Polyphase Representation. With the $k$ th analysis filter written in terms of its $N$ polyphase components $H_{k \mid j}(z), j=0(1) N-1$,

$$
H_{k}(z)=\sum_{j=0}^{N-1} z^{-j} H_{k \mid j}\left(z^{N}\right)
$$

a matrix $\mathbf{H}_{r}(z)$ with polynomial entries can be created for the analysis filter bank:

$$
\mathbf{H}(z)=\left[\begin{array}{cccc}
H_{0 \mid 0}(z) & H_{0 \mid 1}(z) & \cdots & H_{0 \mid N-1}(z) \\
H_{1 \mid 0}(z) & H_{1 \mid 1}(z) & & H_{1 \mid N-1}(z) \\
\vdots & & \ddots & \vdots \\
H_{K-1 \mid 0}(z) & H_{K-1 \mid 1}(z) & \cdots & H_{K-1 \mid N-1}(z)
\end{array}\right]
$$


With a polyphase decomposition of the input signal $x[n]$,

$$
X(z)=\sum_{j=0}^{N-1} z^{-j} X_{j}\left(z^{N}\right)
$$

analogue to (2), and

$$
\underline{X}(z)=\left[X_{0}(z), X_{1}(z), \ldots X_{N-1}(z)\right]^{T}
$$

the analysis bank operation denotes as

$$
\underline{Y}(z)=\mathbf{H}(z) \cdot \underline{X}(z),
$$

where $\underline{Y}(z) \in \mathbb{C}_{(z)}^{K \times 1}$ contains the $K$ subband signals.

If the polyphase matrix $\mathbf{H}(z)$ is paraunitary, the synthesis of the subband signals may be performed by $\underline{X}(z)=\tilde{\mathbf{H}}(z) \cdot \underline{Y}(z)$, where $\tilde{\mathbf{H}}(z)$ is the hermitian of $\mathbf{H}(z)$ with reversed polynomial entries. The reconstructed fullband signal $\hat{x}[n]$ is given in polyphase representation by $\underline{\hat{X}}(z)$. If we combine analysis and synthesis, i.e. $\underline{\hat{X}}(z)=\tilde{\mathbf{H}}(z) \cdot \mathbf{H}(z) \cdot \underline{X}(z)$, perfect reconstructed is characterized by $\tilde{\mathbf{H}}(z) \cdot \mathbf{H}(z)=z^{-L_{p}+1} c \mathbf{I}, c \in \mathbb{C} /\{0\}$, i.e. $\mathbf{H}(z)$ has to be paraunitary [2].

For real input signals $x[n]$, an efficient implementation omits $K / 2$ subbands,

$$
\underline{\hat{X}}(z)=\operatorname{Re}\left\{\tilde{\mathbf{H}}_{r}(z) \cdot \underline{Y}_{r}(z)\right\}=\operatorname{Re}\left\{\tilde{\mathbf{H}}_{r}(z) \cdot \mathbf{H}_{r}(z) \cdot \underline{X}(z)\right\},
$$

where the subscript $r$ refers to a reduced matrix representations including only the upper $K / 2$ rows of $\mathbf{H}(z)$.

Polyphase Factorization. Let, $M$ be the least common multiple (lcm) of the periodicity of the transform in (1), $2 K$, and the decimation ratio $N$, $M=\operatorname{lcm}(2 K, N)$, with $M=J \cdot 2 K=L \cdot N, \quad J, L \in \mathbb{Z}$. To exploit common calculations between filters, the polyphase components of the analysis filters $\mathbf{H}(z)$ can be written in terms of the $M$ polyphase components of the prototype filter $P(z)=\sum_{m=0}^{M-1} z^{-m} P_{m}\left(z^{M}\right)$,

$$
H_{k \mid n}(z)=\sum_{l=0}^{L-1} z^{-l} \cdot t_{k, l N+n} \cdot P_{l N+n}\left(z^{L}\right) .
$$

If the periodicity $2 K$ of the transform coefficients $t_{k, n}$ is considered, it is possible to formulate a dense matrix notation

$$
\mathbf{H}_{r}(z)=\mathbf{T}_{\mathrm{GDFT}, r} \cdot \mathbf{P}(z)
$$

analogue to [2], with the upper half of a GDFT matrix $\mathbf{T}_{\mathrm{GDFT}, r} \in \mathbb{C}^{K / 2 \times 2 K}$ and a generally sparse matrix $\mathbf{P}(z) \in \mathbb{R}_{(z)}^{2 K \times N}$ with $M$ non-zero polynomial 
entries

$$
\mathbf{P}(z)=\left[\mathbf{I}_{2 K} \ldots \mathbf{I}_{2 K}\right] \cdot \operatorname{diag}\left\{P_{0}\left(z^{L}\right), P_{1}\left(z^{L}\right), \ldots P_{M-1}\left(z^{L}\right)\right\} \cdot\left[\begin{array}{c}
\mathbf{I}_{N} \\
z^{-1} \mathbf{I}_{N} \\
\vdots \\
z^{-L+1} \mathbf{I}_{N}
\end{array}\right] .
$$

The GDFT transform matrix $\mathbf{T}_{\mathrm{GDFT}, r}$ in (9) can be further factorized to yield

$$
\mathbf{T}_{\mathrm{GDFT}, r}=\mathbf{D}_{1} \cdot \mathbf{T}_{\mathrm{DFT}, r} \cdot\left[\mathbf{I}_{K} \mathbf{I}_{K}\right] \cdot \mathbf{D}_{2}
$$

where $\mathbf{D}_{1}=e^{j \frac{\pi}{K} k n_{0}} \cdot \mathbf{I}_{K / 2}$ applies a phase correction and $\mathbf{D}_{2} \in \mathbb{C}^{2 K \times 2 K}$ is a diagonal matrix with elements $e^{j \frac{\pi}{K} k_{0}\left(n-n_{0}\right)}, n=0(1) 2 K-1$, introduces the required frequency offset. The representation in (11) allows savings, as $\mathbf{T}_{\mathrm{DFT}, r} \in \mathbb{C}^{K / 2 \times K}$ consists of the upper $K / 2$ rows of a $K$-point DFT matrix with entries $e^{j \frac{2 \pi}{K} k n}$, which can be implemented using standard FFT algorithms.

Computational Complexity. Using this polyphase decomposition and factorization to efficiently implement an analysis bank operation, the signal is processed by a real valued polyphase network, $\mathrm{P}(z)$, followed by a complex transformation. On the synthesis side, the subband signals are rotated by a transform, and only their real part again fed into a polyphase network $\tilde{\mathbf{P}}(z)$. Recording computations, both operations result in a computational complexity of

$$
C_{\text {bank }}=\frac{1}{N}\left(4 K \log _{2} K+6 K+L_{p}\right)
$$

real multiplications per fullband sample.

Extensions. A further reduction compared to (12) can be obtained by a modification of the prototype filter [16]. This reduces the periodicity of the GDFT transform to $K$, thus potentially decreasing the number of polyphase components in $\mathbf{P}(z)$ by a factor 2 , and saves $2 K / N$ multiplications over (12) in the transform evaluation.

\section{Complex Vs Real Valued Subband Processing}

The ratio of computational complexity between adaptive filtering with real and complex valued subband signals for order $\mathcal{O}\left(L_{a}^{i}\right)$ algorithms can be derived as

$$
\begin{gathered}
C_{\text {real }} \propto L_{a}^{i} \quad, \quad C_{\text {complex }} \propto 4 \cdot\left(\frac{1}{2} \cdot\left(\frac{L_{a}}{2}\right)^{i}\right) \\
\longrightarrow \frac{C_{\text {complex }}}{C_{\text {real }}}=\frac{1}{2^{i-1}} .
\end{gathered}
$$




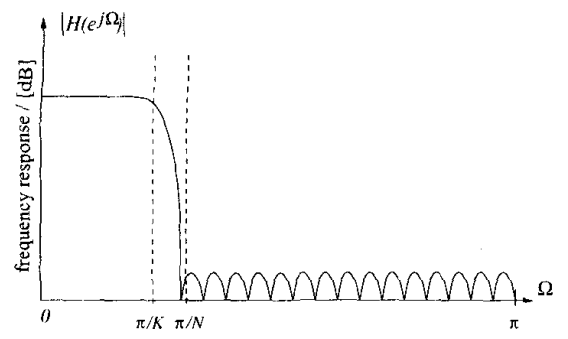

Fig. 3: Required frequency response of a real valued prototype filter $p[n]$ for a $K / 2$ channel oversampled modulated GDFT filter bank with decimation by $N$.

where $L_{a}$ is the length of an adaptive filter. Note that the complex case effectively appears with a doubled decimation ratio, resulting in shorter filters and slower update, but requires 4 real multiplications for a complex one. Thus in terms of processing load, order $\mathcal{O}\left(L_{a}\right)$ algorithms like LMS and NLMS have same computational complexity for complex and real valued implementations, while for quadratic dependencies $(i=2)$ like the RLS the computational burden can be halved by going complex.

Complex subband processing also doubles the range of possible decimation ratios to choose from $\mathbb{N} \ni N \leq K$ over real valued methods like SSB [1] or non-uniform filter banks [6].

\section{PROTOTYPE DESIGN}

This section discusses two requirements of the filter bank design, stopband attenuation and perfect reconstruction, which will both be expressed in terms of the prototype filter. Based on this, an iterative least-squares design is presented.

Stopband Energy. The frequency response of a prototype filter $p[n]$ for a $K$-channel GDFT bank with decimation by $N$ is shown in Fig. 3 . Every frequency of the input signal in the interval $[\pi / N ; \pi]$ will be aliased into the baseband after filtering and decimation, and cause a distortion of the subband signal, which can be modelled as additive noise, motivating an SNR measure for white Gaussian input signals [15]:

$$
\mathrm{SNR}=\frac{\int_{0}^{\pi / N}\left|P\left(e^{j \Omega}\right)\right|^{2} d \Omega}{\int_{\pi / N}^{\pi}\left|P\left(e^{j \Omega}\right)\right|^{2} d \Omega} .
$$

The denominator of (14) forms a measure of the stopband energy. It can be numerically approximated using the Eigenfilter method [13], which evaluates the real part of the frequency response in the stopband

$$
\operatorname{Re}\left\{P\left(e^{j \underline{\Omega}}\right)\right\}=\mathbf{T}_{\mathrm{DCT}} \cdot \mathbf{p}
$$

at discrete frequencies $\Omega_{m}, m=1(1) M$ by multiplying the coefficient vector of the prototype, $\mathbf{p} \in \mathbb{R}^{L_{p} \times 1}$, to a DCT matrix T $\mathrm{T}_{\mathrm{DCT}} \in \mathbb{R}^{M \times L_{p}}$ with entries $t_{m, n}=\cos \left(n \cdot \Omega_{m}\right), n=0(1) L_{p}-1$. 
Symmetry of $\mathbf{p}$ is enforced by introducing a permutation matrix $\mathbf{S}_{1}=$ $\left[\mathbf{I}_{L_{p} / 2}, \mathbf{J}_{L_{p} / 2}\right]$, where $\mathbf{I}$ and $\mathbf{J}$ are identity and the reverse identity matrices and $\mathbf{b}=\mathbf{S}_{1}^{T} \cdot \mathbf{p}$, yielding

$$
\operatorname{Re}\left\{P\left(e^{j \underline{\Omega}}\right)\right\}=\mathbf{T}_{\mathrm{DCT}} \cdot \mathbf{S}_{1} \mathbf{S}_{1}^{T} \cdot \mathbf{p}=\mathbf{T}_{\mathrm{DCT}} \cdot \mathbf{S}_{1} \cdot \mathbf{b} .
$$

Power Complementary Condition. If aliasing is sufficiently suppressed, time-invariance of the input-output behaviour of the filter bank system in Fig. 2 is ensured and the near PR condition reduces to the requirement of power complementarity [5, 13],

$$
\sum_{k=0}^{K-1} H_{k}^{*}\left(z^{-1}\right) \cdot H_{k}(z) \stackrel{!}{=} z^{-L_{p}+1} .
$$

Exploiting the modulation of the passband filters $H_{k}(z)$, this requirement is equivalent to demanding

$$
\sum_{k=0}^{K-1} P_{k}^{*}\left(z^{-1}\right) \cdot P_{k}(z) \stackrel{!}{=} \frac{1}{K} \cdot z^{-L_{p}+1},
$$

where $P_{k}(z)$ are the $K$ polyphase components of the prototype filter $P(z)$ [13]. If the summands on the left hand side are implemented in the time domain by multiplication of a convolutional matrix $\mathbf{P}_{k} \in \mathbb{R}^{(2 L p / K-1) \times\left(L_{p} / K\right)}$ with a vector $\mathbf{p}_{k} \in \mathbb{R}^{(L p / K \times 1)}$ containing the elements of the $k$ th polyphase filter of $p[n],(18)$ becomes

$$
\underbrace{\left[\mathbf{P}_{0} \mathbf{P}_{1} \ldots \mathbf{P}_{K-1}\right]}_{\mathbf{V}} \cdot \underbrace{\left[\begin{array}{c}
\mathbf{p}_{0} \\
\mathbf{p}_{1} \\
\vdots \\
\mathbf{p}_{K-1}
\end{array}\right]}_{\mathbf{v}}=\mathbf{V S}_{2} \cdot \mathbf{p}=\mathbf{V S _ { 2 } \mathbf { S } _ { 1 } \cdot \mathbf { b } \stackrel { ! } { = } [ \begin{array} { c } 
{ \underline { 0 } } \\
{ 1 / K } \\
{ \underline { 0 } }
\end{array} ]}
$$

with a suitable permutation matrix $\mathbf{S}_{2} \in \mathbb{N}^{L_{p}} \times L_{p}$ that maps the coefficient vector $\mathbf{p}$ onto the polyphase vector $\mathbf{v}=\mathbf{S}_{2} \mathbf{p}$.

If the subband adaptive filter is free of any other disturbances, the perfect reconstruction error (PRE)

$$
\mathrm{PRE}=\left\|\mathbf{V S _ { 2 }} \cdot \mathbf{p}-\mathbf{d}\right\|_{2}^{2}
$$

gives a limit measure of the achievable accuracy of the equivalent fullband model at the Wiener-Hopf solution [15].

Iterative Least Squares Design. To fulfill power complementarity and minimize stopband energy, a least-squares problem

$$
\mathbf{b}=\arg \min _{\mathbf{b}}\left\|\left[\begin{array}{c}
\mathbf{V}(\mathbf{b}) \cdot \mathbf{S}_{2} \cdot \mathbf{S}_{1} \\
\gamma \cdot \mathbf{T}_{\mathrm{DCT}} \cdot \mathbf{S}_{1}
\end{array}\right] \mathbf{b}-\left[\begin{array}{l}
\mathbf{d} \\
\underline{0}
\end{array}\right]\right\|_{2}^{2}
$$

has to be solved, where $\gamma$ allows a weighting between both design criteria. The minimization can be performed iteratively $[9,6]$, solving at each iteration 


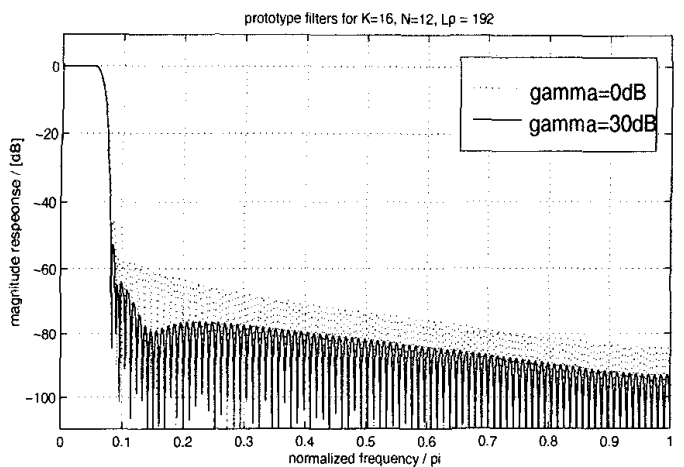

Fig. 4: Frequency responses of prototype filters for $K=16$ channel filter bank with decimation by $N \leq 12$ for two differently balanced designs.

\begin{tabular}{|c||c|c||c|c|}
\hline \multicolumn{4}{|c||}{ Prototype Design } & \multicolumn{2}{c|}{ Simulation Results } \\
\hline$\gamma$ & PRE & SNR & $\|w-s\|_{2}^{2}$ & $\sigma_{d d}^{2} / \sigma_{e e}^{2}$ \\
\hline 0 & -54.0821 & 54.9 & -54.0153 & 54.0 \\
\hline 30 & -34.6191 & 65.2 & -34.6143 & 66.2 \\
\hline 60 & -18.0016 & 77.8 & -18.0010 & 78.5 \\
\hline
\end{tabular}

Tab. 1: first three columns: weighting of design criteria and achieved measures according to (20) and (14); right columns: final equivalent fullband model error and reduction in error power for RLS adaptive identification of a delay (all quantities in $[\mathrm{dB}]$ ).

$k$

$$
\mathbf{b}_{k}=\arg \min _{\mathbf{b}_{k}}\left\|\left[\begin{array}{c}
\mathbf{V}\left(\mathbf{b}_{k-1}\right) \cdot \mathbf{S}_{2} \cdot \mathbf{S}_{1} \\
\gamma \cdot \Lambda_{\omega} \cdot \mathbf{T}_{\mathrm{DCT}} \cdot \mathbf{S}_{1}
\end{array}\right] \mathbf{b}_{k}-\left[\begin{array}{c}
\mathbf{d} \\
\underline{0}
\end{array}\right]\right\|_{2}^{2},
$$

where a previous solution $\mathbf{b}_{k-1}$ is substituted to achieve a quadratic approximation of (21). The resulting problem (22) can be easily solved using standard linear algebraic tools [4]. The iteration is stopped when the change from $\mathbf{b}_{k-1}$ to $\mathbf{b}_{k}$ is below a certain threshold.

Additionally, a diagonal weighting matrix $\Lambda_{\omega}$ in (22) can be included to improve the stopband attenuation towards the band-edge. Furthermore, a relaxation can be introduced to solve for an intermediate solution $\mathbf{b}_{k}^{\prime}$ at step $k$, from which the final solution is obtained by $\mathbf{b}_{k}=\alpha \mathbf{b}_{k}^{\prime}+(1-\alpha) \mathbf{b}_{k-1}$, for $0<\alpha \leq 1$.

\section{RESULTS}

Design Examples. Fig. 4 shows two prototype filters obtained using the above iterative LS design. The starting coefficients $\mathbf{b}_{0}$ are calculated by a remez filter design, and for a relaxation $\alpha=0.5$ the discussed LS method requires 11 iterations to converge in $8.44 \mathrm{~s} \mathrm{CPU}$ time on a Sparc20 workstation. The final PRE and SNR values of the design are listed in Tab. 1. 


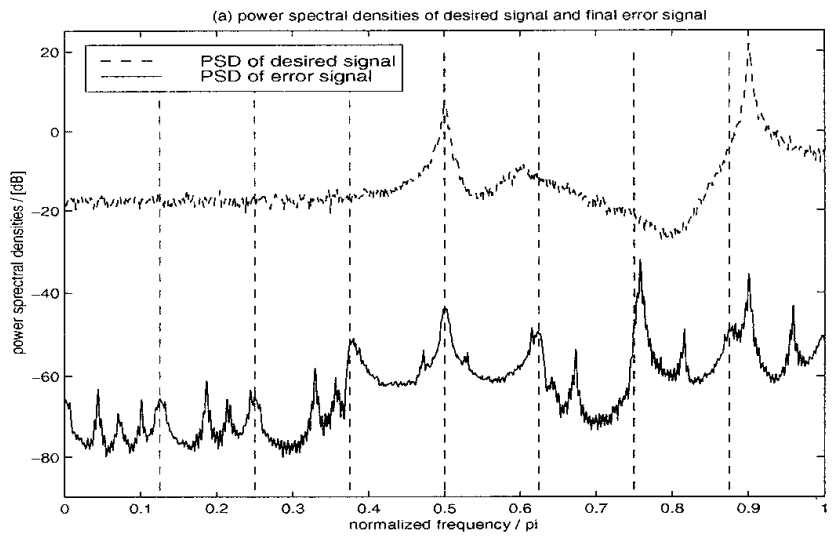

Fig. 5: PSD of desired signal and final error signal; dashed vertical lines indicate the band edges.

Subband Adaptive Filtering Simulations. Using the prototype filters designed in Tab. 1, adaptive system identification of a white Gaussian noise excited delay was performed in subbands. The error terms after convergence of the adaptive filters using the RLS algorithm [7] are also shown in Tab. 1 and closely agree with the properties calculated from the prototype design. Using the prototype for $\gamma=0 d B$, Fig. 5 shows simulation results employing an NLMS algorithm for adaptive identification of an unknown system with several dominant poles. Besides insufficient convergence at the band edges, and residual peaks and a raised error power spectrum around the positions of the poles, one can clearly see the aliased peaks of the desired signal, which limit adaptation. The achieved reduction in error power is $52.92 \mathrm{~dB}$, while the $l_{2}$ distance of the reconstructed equivalent fullband response, $w[n]$, from to the unknown system, $s[n]$, is $-50.43 \mathrm{~dB}$, well matching the design measures in Tab. 1 .

\section{CONCLUSIONS}

We have shown that GDFT filter banks can enable highly efficient subband adaptive filter schemes, by extending the polyphase realization of the filter banks to the case of general integer decimation ratios, and by the very nature of complex subband decompositions of real valued input signals. Furthermore, based on the prototype filter, a fast converging design method has been discussed, which minimizes two criteria limiting the performance of a subband adaptive system. The appeal is that these criteria provide convenient tools to design filter banks fulfilling pre-specified, application dependent performance requirements. For applications like acoustic echo control, where the adaptation error is the most important issue, the banks can be designed to be just good (and short) enough to satisfy relaxed constraints on the model error. 


\section{References}

[1] R. E. Crochiere and L. R. Rabiner. Multirate Digital Signal Processing. Prentice Hall, 1983.

[2] Z. Cvetković and M. Vetterli. "Oversampled Filter Banks". IEEE Trans. Signal Processing, SP-46(5):1245-1259, 1998.

[3] A. Gilloire and M. Vetterli. "Adaptive Filtering in Subbands with Critical Sampling: Analysis, Experiments and Applications to Acoustic Echo Cancelation". IEEE Trans. Signal Processing, SP-40(8):1862-1875, 1992.

[4] G. H. Golub and C. F. Van Loan. Matrix Computations. Johns Hopkins University Press, 1996.

[5] M. Harteneck, J. M. Páez-Borrallo, and R. W. Stewart. "An Oversampled Subband Adaptive Filter Without Cross Adaptive Filters". Signal Processing, to appear, 1998.

[6] M. Harteneck and R. W. Stewart. "Filterbank Design for Oversampled Filter Banks Without Aliasing in the Subbands". IEE Electr. Lett., 38(18):1538-1539, 1997.

[7] S. Haykin. Adaptive Filter Theory. Prentice Hall, 1991.

[8] Q.-G. Liu and B. Champagne. "Simple Design of Filter Banks for Subband Adaptive Filtering". Proc. IWAENC, pp:132-135, London, 1997.

[9] M. Rossi, J.-Y. Zhang, and W. Steenaart. "A New Algorithm for Designing Prototype Filters for M-Band Pseudo QMF Banks". In Proc. EUSIPCO, II:1195-1198, Trieste, 1996.

[10] J. J. Shynk. "Frequency-Domain and Multirate Adaptive Filtering". IEEE Signal Processing Magazine, 9:14-37, Jan. 1992.

[11] V. Somayazulu, S. Mitra, and J. Shynk. "Adaptive Line Enhancement Using Multirate Techniques". In Proc. IEEE International Conference on Acoustics, Speech, and Signal Processing, volume 2, pages 928-931, Glasgow, Scotland, UK, May 1989.

[12] O. Tanrikulu, B. Baykal, A. G. Constantinides, and J. Chambers. "Residual Echo Signal in Critically Sampled Subband Acoustic Echo Cancellers Based on IIR and FIR Filter Banks". IEEE Trans. Signal Processing, 45(4):901-912, 1997.

[13] P. P. Vaidyanathan. Multirate Systems and Filter Banks. Prentice Hall, 1993.

[14] S. Weiß, L. Lampe, and R. W. Stewart. "Efficient Implementations of Complex and Real Valued Filter Banks for Comparative Subband Processing with an Application to Adaptive Filtering". To appear in Proc. Int. Symp. Comms Systems \& Digital Signal Processing, Sheffield, England, April 1998.

[15] S. Weiß, R. W. Stewart, A. Stenger, and R. Rabenstein. "Performance Limitations of Subband Adaptive Filters". To appear in Proc. EUSIPCO, Rodos, Greece, 1998.

[16] S. Weiß. "On Adaptive Filtering on Oversampled Subbands". PhD dissertation, Signal Processing Division, University of Strathclyde, Glasgow, May 1998.

[17] Y. Yamada, H. Ochi, and H. Kiya. "A Subband Adaptive Filter Allowing Maximally Decimation". IEEE J. Selected Areas Comms, 12(9):1548-1552, 1994. 